\title{
SEPARABILITY OF THE L'-SPACE OF A VECTOR MEASURE
}

\author{
by WERNER J. RICKER
}

(Received 22 June, 1990)

Let $\Sigma$ be a $\sigma$-algebra of subsets of some set $\Omega$ and let $\mu: \Sigma \rightarrow[0, \infty]$ be a $\sigma$-additive measure. If $\Sigma(\mu)$ denotes the set of all elements of $\Sigma$ with finite $\mu$-measure (where sets equal $\mu$-a.e. are identified in the usual way), then a metric $d$ can be defined in $\Sigma(\mu)$ by the formula

$$
d(E, F)=\mu(E \Delta F)=\int_{\Omega}\left|\chi_{E}-\chi_{F}\right| d \mu \quad(E, F \in \Sigma) ;
$$

here $E \Delta F=(E \backslash F) \cup(F \backslash E)$ denotes the symmetric difference of $E$ and $F$. The measure $\mu$ is called separable whenever the metric space $(\Sigma(\mu), d)$ is separable. It is a classical result that $\mu$ is separable if and only if the Banach space $L^{1}(\mu)$ is separable [8, p. 137]. To exhibit non-separable measures is not a problem; see [8, p. 70], for example. If $\Sigma$ happens to be the $\sigma$-algebra of $\mu$-measurable sets constructed (via outer-measure $\mu^{*}$ ) by extending $\mu$, defined originally on merely a semi-ring of sets $\Gamma \subseteq \Sigma$, then it is also classical that the countability of $\Gamma$ guarantees the separability of $\mu$ and hence, also of $L^{1}(\mu),[8$, p. 69].

There arises the natural question of what form such classical results on separability of $L^{1}$-spaces should take for vector-valued measures. We aim to formulate such results in this note.

So, suppose that $X$ is a locally convex space (briefly, Ics), always assumed to be Hausdorff and sequentially complete. A $\sigma$-additive map $m: \Sigma \rightarrow X$, where $\Sigma$ is a $\sigma$-algebra of subsets of some set $\Omega$, is called a ( $X$-valued) vector measure. A $\Sigma$-measurable function $f: \Omega \rightarrow \mathbb{C}$ is called $m$-integrable if it is integrable with respect to the complex measure $\left\langle m, x^{\prime}\right\rangle: E \mapsto\left\langle m(E), x^{\prime}\right\rangle$, for $E \in \Sigma$, for every $x^{\prime} \in X^{\prime}$ (the continuous dual space of $X$ ), and if, for every $E \in \Sigma$, there exists an element of $X$, denoted by $\int_{E} f d m$, which satisfies $\left\langle\int_{E} f d m, x^{\prime}\right\rangle=\int_{E} f d\left\langle m, x^{\prime}\right\rangle$, for every $x^{\prime} \in X^{\prime}$. The linear space of all $m$-integrable functions is denoted by $L(m)$. Let $\mathscr{2}_{X}$ denote the family of all continuous seminorms in $X$ or, at least enough seminorms to determine the topology of $X$. Each $q \in \mathscr{2}_{X}$ induces a seminorm $q(m)$ in $L(m)$ via the formula

$$
q(m): f \mapsto \sup \left\{\int_{\Omega}|f| d\left|\left\langle m, x^{\prime}\right\rangle\right| ; x^{\prime} \in U_{q}^{0}\right\} \quad(f \in L(m)),
$$

where $U_{q}^{0} \subseteq X^{\prime}$ denotes the polar of the unit ball $U_{q}=q^{-1}([0,1])$. The seminorms (2), as $q$ varies through $2_{X}$, define a lc topology $\tau(m)$ in $L(m)$. Since $\tau(m)$ may not be Hausdorff we form the usual quotient space of $L(m)$ with respect to the closed subspace $\bigcap_{q \in 2 x} q^{-1}(\{0\})$. The resulting Hausdorff space (with topology again denoted by $\tau(m)$ ) is denoted by $L^{1}(m)$; it can be identified with equivalence classes of functions from $L(m)$ modulo $m$-null functions, where a function $f \in L(m)$ is $m$-null whenever $\int_{E} f d m=0$, for every $E \in \Sigma$. All of the above definitions and further properties of $L^{1}(m)$ can be found in [6].

Let $\Sigma(m)$ denote the subset of $L^{1}(m)$ corresponding to $\left\{\chi_{E} ; E \in \Sigma\right\} \subseteq L(m)$. Of course, elements of $\Sigma(m)$ can also (and will) be identified with equivalence classes of elements from $\Sigma$. The formula (1) suggests how to topologize $\Sigma(m)$. Namely, we restrict 
the $L^{1}(m)$-topology $\tau(m)$ to $\Sigma(m)$. That is, each seminorm $q(m)$, where $q \in \mathscr{Q}_{X}$, induces a semi-metric $d_{q}$ on $\Sigma(m)$ by the formula

$$
d_{q}\left(\chi_{E}, \chi_{F}\right)=q(m)\left(\chi_{E}-\chi_{F}\right) \quad(E, F \in \Sigma) .
$$

Again $\tau(m)$ will denote the uniform structure and topology in $\Sigma(m)$ so defined by the semi-metrics (3) as $q$ varies through $2_{X}$.

1. Main results. Throughout this section $X$ is a Hausdorff, sequentially complete Ics. A vector measure $m: \Sigma \rightarrow X$ is called separable whenever the topological space $(\Sigma(m), \tau(m))$ is separable. For $X=\mathbb{C}$ (or $\mathbb{R}$ ) this coincides with the classical definition.

Proposition 1. Let $m: \Sigma \rightarrow X$ be a vector measure.

(i) If the measure $m$ is separable, then the lcs $L^{1}(m)$ is separable.

(ii) Let the lcs $X$ be metrizable. Then $m$ is separable if and only if $L^{1}(m)$ is separable.

Proof. (i) Let $B \subseteq \Sigma(m)$ be a countable $\tau(m)$-dense set in $\Sigma(m)$. Then the collection $\mathscr{S}(B)$ of all simple functions of the form $\sum_{j=1}^{k} \alpha_{j} \chi_{F(j)}$ for $k$ a positive integer, $\alpha_{j}$ a "rational complex number" and $F(j) \in B, 1 \leq j \leq k$, is also countable. By the $\tau(m)$-density of the $\Sigma$-simple functions in $L^{1}(m),[6, \mathrm{Ch} .2]$, it suffices to show that if $f=\sum_{j=1}^{n} \beta_{j} \chi_{E(j)}$ is a $\Sigma$-simple function and positive numbers $\epsilon_{r}$ are given together with seminorms $q_{r} \in \mathscr{2}_{X}$, $1 \leq r \leq k$, then there exists an element $h \in \mathscr{S}(B)$ satisfying

$$
q_{r}(m)(f-h)<\epsilon_{r} \quad(1 \leq r \leq k) .
$$

Let $\epsilon=\min \left\{\epsilon_{r} ; 1 \leq r \leq k\right\}$ and $K=\max \left\{q_{r}(m)\left(\chi_{\Omega}\right) ; 1 \leq r \leq k\right\}$. Choose "rational complex numbers" $\alpha_{j}, 1 \leq j \leq n$, satisfying $\left|\alpha_{j}-\beta_{j}\right|<\epsilon /(2 n K)$ for $1 \leq j \leq n$. By $\tau(m)$ density of $B$ in $\Sigma(m)$ there exist sets $F(j) \in B$ such that, for every $j \in\{1,2, \ldots, n\}$ we have

$$
d_{q_{r}}(E(j), F(j))=q_{r}(m)\left(\chi_{E(j)}-\chi_{F(j)}\right)<\epsilon_{j} /(2 n \beta),
$$

for every $1 \leq r \leq k$, where $\beta=\max \left\{\left|\beta_{j}\right| ; 1 \leq j \leq n\right\}$. Let $h$ be the element $\sum_{j=1}^{n} \alpha_{j} \chi_{F(j)}$ of $\mathscr{S}(B)$. Since

$$
|f-h| \leq \sum_{j=1}^{n}\left|\alpha_{j}-\beta_{j}\right| \chi_{F(j)}+\sum_{j=1}^{n}\left|\beta_{j}\right| \cdot\left|\chi_{F(j)}-\chi_{E(j)}\right|
$$

it follows that

$$
|f-h| \leq \epsilon(2 n K)^{-1} \sum_{j=1}^{n} \chi_{F(j)}+\beta \sum_{j=1}^{n}\left|\chi_{F(j)}-\chi_{E(j)}\right| .
$$

Since $q_{r}(m)\left(\chi_{F(j)}\right) \leq q_{r}(m)\left(\chi_{\Omega}\right)$, for every $1 \leq j \leq n$ and $1 \leq r \leq k$, and $q(m)(g)=$ $q(m)(|g|)$, for every $q \in \mathscr{Q}_{X}$ and $g \in L^{1}(m)$-see (2)-it follows from (5), (6) and the definitions of $K$ and $\epsilon$ that (4) is satisfied.

(ii) If $X$ is metrizable, then $2_{X}$ can be chosen to be a countable set. It is then clear from the definition of $\tau(m)$ that $L^{1}(m)$ is also a metrizable lcs. Since $(\Sigma(m), \tau(m))$ is a subset of $L^{1}(m)$ with the relative topology it follows that $(\Sigma(m), \tau(m))$ is separable whenever $L^{1}(m)$ is separable [8, p. 20]. 
It would seem useful to have available a criterion for determining separability. Given a measure $m: \Sigma \rightarrow X$ we recall that $\Sigma$ is called $m$-essentially countably generated [6, p. 32] if there exists a countably generated $\sigma$-algebra $\Sigma_{0} \subseteq \Sigma$ such that $\Sigma(m)=\Sigma_{0}(m)$.

PropostTion 2. Let $m: \Sigma \rightarrow X$ be a vector measure. If $\Sigma$ is m-essentially countably generated, then $m$ is a separable measure. In particular, $L^{\prime}(m)$ is separable.

The proof of this result relies on the following two facts: the first is straightforward and the second follows from the first and [3, III Lemma 8.4].

Lemma 1. (i) Let $\Lambda$ be a family of subsets of a set $\Omega$. Then the $\sigma$-algebras of subsets of $\Omega$ generated by $\Lambda \cup\{\Omega\}$ and by $\Lambda$ coincide.

(ii) Let $\Sigma$ be a countably generated $\sigma$-algebra of subsets of a set $\Omega$. Then there exists a countable algebra of sets $\Sigma_{0} \subseteq \Sigma$ such that the o-algebra generated by $\Sigma_{0}$ is precisely $\Sigma$.

Proof of Proposition 2. Let $\Sigma_{0}$ be a countable algebra of subsets of $\Omega$ which $m$-essentially generates $\Sigma$. Let $m_{0}$ denote the restriction of $m$ to $\Sigma_{0}$. Then $m_{0}$ is $\sigma$-additive on $\Sigma_{0}$ and has an extension to a $\sigma$-additive measure on $\Sigma$, namely $m$. It follows from the equivalence of (i) and (xi) in the Theorem of Extension in [5] (the topology $\tau^{*}(m)$ stated there in (xi) coincides with our $\tau(m)$; see p. 178 of [5]) that $\Sigma_{0}$ is $\tau(m)$-dense in $\Sigma \simeq \Sigma(m)$. Accordingly, $m$ is separable.

Corollary 1. Let $m: \Sigma \rightarrow X$ be a vector measure. If $\Sigma$ is m-essentially countably generated, then the closed subspace of $X$ generated by the range of $m$ is separable for the relative topology induced by $X$.

Proof. The integration map $\Phi$ given by $\Phi: f \mapsto \int_{\Omega} f d m$, for $f \in L^{1}(m)$, is continuous from $\left(L^{1}(m), \tau(m)\right)$ into $X$. Let $Y$ denote the closed subspace of $X$ generated by the range, $m(\Sigma)=\{m(E) ; E \in \Sigma\}$, of $m$. By approximating elements of $L^{1}(m)$ by $\Sigma$-simple functions it is clear that $\Phi\left(L^{1}(m)\right) \subseteq Y$ and hence, the closure $\overline{\Phi\left(L^{1}(m)\right)} \subseteq Y$. But, the formula $m(E)=\Phi\left(\chi_{E}\right)$, for $E \in \Sigma$, shows that actually $\overline{\Phi\left(L^{1}(m)\right)}=Y$.

The proof of Proposition 2 showed that there exists a countable algebra of sets $B$ which $m$-essentially generates $\Sigma$ and such that $B$ is $\tau(m)$-dense in $\Sigma(m)$. Then the collection $\mathscr{S}(B)$ of "rational" $B$-simple functions as defined in the proof of Proposition 1 (i) is countable and dense in $L^{1}(m)$. Clearly the $\Phi$-image of the set $\mathscr{Y}(B)$ is countable and contained in $Y$. So, it suffices to show that elements of $\Phi\left(L^{1}(m)\right)$ can be approximated (in $X$ ) by elements of $\Phi(\mathscr{S}(B)$ ). That this is the case follows from the density of $\mathscr{S}(B)$ in $L^{1}(m)$ and the continuity of $\Phi$.

In many situations, the converse of Proposition 2 is also valid. In order to formulate it we recall some notions from topology. Let $\Lambda$ be a topological Hausdorff space and $Y \subseteq \Lambda$. Then $[Y]$ denotes the set of all elements in $\Lambda$ which are the limit of some sequence of points from $Y$. A set $Y \subseteq \Lambda$ is called sequentially closed if $Y=[Y]$. The sequential closure $\bar{Y}_{s}$, of a set $Y \subseteq \Lambda$, is the smallest sequentially closed subset of $\Lambda$ which contains $Y$. Alternatively, let $Y_{0}=Y$. Let $\Omega_{1}$ be the smallest uncountable ordinal. Suppose that $0<\alpha<\Omega_{1}$ and that $Y_{\beta}$ has been defined for all ordinals $\beta$ satisfying $0 \leq \beta<\alpha$. Define $Y_{\alpha}=\left[\bigcup_{0 \leq \beta<\alpha} Y_{\beta}\right]$. Then $\bar{Y}_{s}=\bigcup_{0 \leq \alpha<\Omega_{1}} Y_{\alpha}$.

Let $\mathscr{A}$ be a family of subsets of a non-empty set $\Omega$. Then the $\sigma$-algebra of subsets generated by $\mathscr{A}$ is denoted by $\mathscr{A}_{\sigma}$. The cardinality of a set $B$ is denoted by $\#(B)$. 
Lemma 2. (i) Let $\mathscr{A}$ be an infinite family of subsets of a non-empty set $\Omega$. Then the algebra of sets generated by $\mathscr{A}$ has cardinality $\#(\mathscr{A})$. Moreover, $\#\left(\mathscr{A}_{\sigma}\right) \leq \#(\mathscr{A})^{\mathrm{x}_{0}}$.

(ii) If $Y$ is a subset of a topological space $\Lambda$, then $\#\left(\bar{Y}_{s}\right) \leq \#(Y)^{\aleph_{0}}$.

(iii) Let $m: \Sigma \rightarrow X$ be a vector measure and $\mathscr{A} \subseteq \Sigma$ be an algebra of sets. Then $\chi\left(\mathscr{A}_{\sigma}\right)=\left\{\chi_{E} ; E \in \mathscr{A}_{\sigma}\right\}$ is contained in the sequential closure of $\chi(\mathscr{A})$ in the topological space $\Sigma(m)$.

Proof. (i) This can be found on pp. 133-134 of [4].

(ii) follows from the transfinite inductive definition of $\bar{Y}_{s}$ and a modification of the proof of Theorem 10.23 in [4].

(iii) Recall that $\mathscr{A}_{\sigma}$ can be constructed as follows (see [5, p. 180], for example): beginning with $\mathscr{A}$, let $\mathscr{A}_{i}$ be the system of all sets expressible as the union of increasing sequences of elements from $\mathscr{A}$; then construct the system $\mathscr{A}_{\text {id }}$ of intersections of decreasing sequences in $\mathscr{A}_{i}$, then construct $\mathscr{A}_{i d i}$, and so on by transfinite induction all the way to $\Omega_{1}$. At each stage of this procedure the monotone convergence theorem for $m$, $[6, \mathrm{Ch} . \mathrm{II}, \S 4]$, guarantees that the next family of sets belongs to $\overline{\chi(\mathscr{A})_{s}}$.

We can now formulate a partial converse to Proposition 2 which is applicable to a large class of vector measures; see Remark 1 below.

PROPOSITION 3. Let $m: \Sigma \rightarrow X$ be a vector measure such that its range $m(\Sigma)$ is metrizable for the relative topology from $X$.

(i) Let $\mathscr{A} \subseteq \Sigma$ be an algebra of sets. Then $\mathscr{A}_{\sigma}=\overline{\mathscr{A}}_{s}$, meaning that $\chi\left(\mathscr{A}_{\sigma}\right)$ and $\overline{\chi(\mathscr{A})}$, coincide as subsets of $\Sigma(m)$. In particular, $\bar{A}_{s}$ is a $\sigma$-algebra of sets.

(ii) The measure $m$ is separable if and only if $\Sigma$ is m-essentially countably generated.

Proof. (i) The inclusion $\mathscr{A}_{\sigma} \subseteq \overline{\mathscr{A}}_{s}$ follows from Lemma 2(iii). To establish the reverse inclusion it suffices to show that $\mathscr{A}_{\sigma}$ is sequentially closed. So, let $E(n)$, $n=1,2, \ldots$, be elements of $\mathscr{A}_{\sigma}$ such that $\chi_{E(n)} \rightarrow f$ in $L^{1}(m)$. Since $\Sigma(m)$ is $\tau(m)$ complete by [7, Proposition 1], it follows that $f=\chi_{E}$ for some $E \in \Sigma$. An examination of the proof of Proposition 1 in [7] shows that there exists a sequence of continuous seminorms $\left\{q_{k}\right\}_{k=1}^{\infty}$ in $X$ such that corresponding semi-metrics $\left\{d_{q_{k}}\right\}_{k=1}^{\infty}$ given by (3) induce the metrizable topology on $m(\Sigma)$. Arguing as in the proof of [6, II Section 1 , Corollary 2] it follows that there exists a finite positive measure $\lambda$ on $\Sigma$, with the same null sets as $m$, satisfying $\lambda(F) \rightarrow 0$ whenever $q_{k}(m)(F) \rightarrow 0$ for each $k=1,2, \ldots$ Accordingly, $\lambda(E(n) \Delta E) \rightarrow 0$ as $n \rightarrow \infty$. Then there is a subsequence $\left\{E\left(n_{r}\right)\right\}_{r=1}^{\infty}$ of $\{E(n)\}_{n=1}^{\infty}$ such that $\chi_{E\left(n_{r}\right)} \rightarrow \chi_{E}, \lambda$-a.e. and hence $m$-a.e. It follows that $E \in \mathscr{A}_{\sigma}$.

(ii) One direction is clear from Proposition 2. Conversely, suppose that $m$ is separable. Then $\Sigma(m)$ has a countable dense set. By Lemma 2(i) the algebra of sets that it generates, say $\mathscr{A}$, is also countable (and still dense). By part (i), $\mathscr{A}_{\sigma}=\overline{\mathscr{A}}_{s}$. Since $\Sigma(m)$ is metrizable the sequential closure of $\mathscr{A}$ coincides with its $\tau(m)$-closure. Accordingly, $\Sigma(m)=\mathscr{A}_{\sigma}$ and so $\Sigma(m)$ is $m$-essentially countably generated.

Remark 1. Many lc spaces $X$, themselves not necessarily metrizable, have the property that their bounded sets are metrizable; see [7], for example. In such spaces, every vector measure $m: \Sigma \rightarrow X$ necessarily has metrizable range and hence is separable if and only if $\Sigma$ is $m$-essentially countably generated. 
Remark 2. An essential ingredient in the proof of Proposition 3(i) was the existence of a finite, non-negative measure $\lambda$ with the same null sets as $m$ and having the property that $\chi_{E(n)} \rightarrow \chi_{E}$ in $\Sigma(m)$ implies $\lambda(E(n) \Delta E) \rightarrow 0$. There are other instances when such a measure $\lambda$ exists without the range $m(\Sigma)$ being metrizable. For example, let $X$ be a Banach space and $L_{s}(X)$ be the space of all continuous linear operators of $X$ into itself, equipped with the strong operator topology. Let $P: \Sigma \rightarrow L_{s}(X)$ be a spectral measure, that is, a $\sigma$-additive measure satisfying $P(\Omega)=I$ (the identity operator on $X$ ) and $P(W \cap F)=P(E) P(F)$, for every $E, F \in \Sigma$. If $X$ is non-separable then, except for trivial cases, $P(\Sigma)$ is not metrizable for the strong operator topology. Suppose that a separating vector $x \in X$ exists for $P$; that is, $P(E) x=0$ implies $P(E)=0$. For example, cyclic vectors are always separating. By a classical result of $W$. Bade [1, Theorem 3.1] there exists $x^{\prime} \in X^{\prime}$ such that the (finite) measure $\lambda=\left\langle P() x,. x^{\prime}\right\rangle$ is non-negative on $\Sigma$ and satisfies $P(E) x=0$ whenever $\lambda(E)=0$. Since $x$ is separating we have $P(E)=0$ if and only if $\lambda(E)=0$. Moreover, if $q(P)(E(n)) \rightarrow 0$, for all continuous seminorms $q$ in $L_{s}(X)$, then also $P(E(n)) \rightarrow 0$ in $L_{s}(X)$ from which it is clear that $\lambda(E(n)) \rightarrow 0$. So, Proposition 3(i) holds for any spectral measure $P$ with a separating vector.

REMARK 3. An essential ingredient in the proof of Proposition 3(ii) was the fact that the sequential closure $\overline{\mathscr{A}}_{s}$ was all of $\Sigma(m)$. Here, the metrizability of $\Sigma(m)$ was used. The following example shows that this condition cannot be removed in general.

EXAMPLE 1. Let $X=\mathbb{C}^{[0.1]}$ denote the vector space of all $\mathbb{C}$-valued functions on $\Omega=[0,1]$ equipped with pointwise operations. For each $\omega \in \Omega$, define a seminorm $q_{\omega}: f \mapsto|f(\omega)|$, for $f \in X$. The seminorms $q_{\omega}(\omega \in \Omega)$ determine a complete Ic Hausdorff topology on $X$. Bounded subsets of $X$ are not necessarily metrizable.

Let $\Sigma$ denote the $\sigma$-algebra of all subsets of $\Omega$. Then the set function $m: \Sigma \rightarrow X$ defined by

$$
m(E)=\chi_{E}, \quad E \in \Sigma,
$$

is $\sigma$-additive. Moreover, every function $\psi: \Omega \rightarrow \mathbb{C}$ belongs to $L^{1}(m)$. Indeed, $\int_{E} \psi d m=$ $\chi_{E} \psi, E \in \Sigma$. It is routine to check that the topology $\tau(m)$ is precisely that of $X$ and hence, $\left(L^{\prime}(m), \tau(m)\right)$ is isomorphic to $X$.

Now, the space $(\Sigma(m), \tau(m))$ can be identified with $\{0,1\}^{\Omega}$ equipped with its product topology. Since $\#(\Omega)=c$ the space $\Sigma(m) \simeq\{0,1\}^{\Omega}$ is separable. Let $\mathscr{A}$ be any countable algebra of sets whose $\tau(m)$ closure is $\Sigma(m)$. By Lemma 2(ii) we have $\#\left(\overline{\chi(\mathscr{A})_{s}}\right) \leq \kappa_{0}^{N_{0}}<$ $\#(\Sigma(m))$ and so the sequential closure $\overline{\mathscr{A}}_{s}$ cannot be all of $\Sigma(m)$.

REMARK 4. Example 1 is of interest for other reasons. We say that an algebra $\mathscr{A}$ of subsets of $\Omega=[0,1]$ separates points of $\Omega$ if, whenever $u$ and $v$ are distinct points of $\Omega$ there is a set $A \in \mathscr{A}$ such that $A \cap\{u ; v\}$ is a singleton. The following observation is straightforward to check.

FACT 1. Let $X$ be the lcs of Example 1. Let $\Sigma$ be any $\sigma$-algebra of subsets of $\Omega=[0,1]$ and $m: \Sigma \rightarrow X$ be the vector measure given by (7). Then $L^{1}(m)$ is precisely the space of all $\mathbb{C}$-valued, $\Sigma$-measurable functions on $\Omega$, equipped with the relative topology from $X$.

(i) If there exists a countable subalgebra of $\Sigma$ which separates points of $\Omega$, then $(\Sigma(m), \tau(m))$ is separable; that is, $m$ is a separable measure. 
(ii) Suppose that $\Sigma(m)$ (which equals $\Sigma$ as there are no non-trivial m-null sets) is $\tau(m)$-separable. If $\Sigma$ contains all finite subsets of $\Omega$, then there is a countable algebra $\mathscr{A} \subseteq \Sigma$ such that $\mathscr{A}$ separates points of $\Omega$.

Using Fact 1 it is clear, when $\Sigma$ is the $\sigma$-algebra of Borel subsets of $\Omega$, or the Lebesgue measurable subsets of $\Omega$, or the universally measurable subsets of $\Omega$, that the measure $m$ given by (7) is always separable and hence, so is $L^{1}(m)$. However, if $\Sigma$ is the $\sigma$-algebra of countable and co-countable subsets of $\Omega$, then it can be shown that $\Sigma$ is not countably generated, $m$ is not separable and $L^{1}(m)$ is not separable (use the fact that if $f \in L^{1}(m)$, then $f$ is constant on the complement of some countable set). Another example of this phenomenon occurs for the measure $P$ of Example 2 below.

A further feature of the class of measures given by (7) is the following observation (which does not follow from Proposition 3(i)).

FACT 2. Let $X$ be the lcs of Example 1. Let $\Sigma$ be any $\sigma$-algebra of subsets of $\Omega=[0,1]$ and $m: \Sigma \rightarrow X$ be the vector measure given by (7). Then the sequential closure $\bar{\Sigma}_{s}$ (taken in $X)$ is actually a $\sigma$-algebra.

Proof. By Lemma 2(iii) it follows that $\Sigma \subseteq \Sigma_{s}$. Conversely, suppose that $\{E(n)\}_{n=1}^{\infty}$ is a sequence of sets from $\Sigma$ which converges to $f$ in $L^{1}(m)$; that is, $\chi_{E(n)} \rightarrow f$ in $\mathbb{C}^{\Omega}$. It is then clear that $f=\chi_{E}$ for some set $E \subseteq \Omega$. But, the pointwise limit of a sequence of $\Sigma$-measurable functions is $\Sigma$-measurable and so $E \in \Sigma$. Continuing this argument via the transfinite inductive definition of $\overline{\boldsymbol{\Sigma}}_{s}$ we conclude that $\overline{\boldsymbol{\Sigma}}_{s} \subseteq \Sigma$.

As a simple consequence, let $\Sigma$ be the Lebesgue measurable sets in [0,1]. With $X$ as in Example 1 and $m: \Sigma \rightarrow X$ given by (7) we have seen that $\Sigma(m)=\Sigma$ is separable. Let $\mathscr{A}$ be a countable algebra of sets in $\Sigma$ whose $\tau(m)$-closure is $\Sigma$. By Fact 2 we note that $\overline{\mathscr{A}}_{s}$ (taken in $X$ ) is a $\sigma$-algebra. However, $\bar{A}_{s} \neq \Sigma$. This follows from Lemma 2(ii) and the fact that $\#(\Sigma)=2^{c}$.

REMARK 5. The results of this section suggest the following two natural questions.

(i) Do there exist a lcs $X$, a measure $m: \Sigma \rightarrow X$ and an algebra of sets $\mathscr{A} \subseteq \Sigma$ such that $\bar{A}_{s}$ is not sequentially closed in $\Sigma(m)$ ?

(ii) Do there exist a lcs $X$ and a measure $m: \Sigma \rightarrow X$ such that $m$ is not a separable measure but $L^{1}(m)$ is separable?

It may be worth noting that Example 1 does not answer Question (ii). For, if $X$ is the lcs given there and $\Sigma$ is any $\sigma$-algebra of subsets of $\Omega=[0,1]$, then $m: \Sigma \rightarrow X$ (given by (7)) is a separable measure if and only if $L^{1}(m)$ is separable. Indeed, suppose that $L^{1}(m)$ is separable. Considering only $\mathbb{R}$-valued functions, let $\mathscr{F} \subseteq L^{1}(m)$ be a countable dense set and, for $f \in \mathscr{F}$, set $E(f)=\left\{\omega \in \Omega ; f(\omega)>\frac{1}{2}\right\}$. It turns out that $D=\left\{\chi_{E(f)} ; f \in \mathscr{F}\right\}$ is a (countable) dense set in $\Sigma(m)$. The case for $\mathbb{C}$-valued functions then follows. The converse claim follows from Proposition 1(i). The fact that $\Omega$ is the interval $[0,1]$ is not important. Indeed, if $\Omega$ is any non-empty set and $X=\mathbb{C}^{\Omega}$ (with the pointwise convergence topology), then a similar argument shows that a vector measure $m: \Sigma \rightarrow X$ (with $\Sigma$ a $\sigma$-algebra of subsets of $\Omega$ ) of the form (7) is separable if and only if $L^{1}(m)$ is separable.

2. Operator-valued measures. Let $X$ be a lcs and $T \in L(X)$ be a scalar-type spectral operator. Such an operator $T$ has a unique (equicontinuous) spectral measure 
$P_{T}: \mathscr{B}(\mathbb{C}) \rightarrow L_{s}(X)$, such that the identity function $\lambda$ (on $\mathbb{C}$ ) is $P_{T}$-integrable and $T=\int_{\mathbb{C}} \lambda d P_{T}$. Here $\mathscr{B}(\mathbb{C})$ is the $\sigma$-algebra of Borel subsets of $\mathbb{C}$. The measure $P_{T}$ is called the resolution of the identity for $T$. So, $L^{1}\left(P_{T}\right)$ is always $\tau\left(P_{T}\right)$-separable. Under certain completeness assumptions on the lc spaces $L_{s}(X)$ and $L^{1}\left(P_{T}\right)$ it turns out that $L^{1}\left(P_{T}\right)$ is isomorphic to the strong operator closed algebra in $L_{s}(X)$ generated by the range of the resolution of the identity for $T$; see [2]. Accordingly, this algebra of operators is necessarily separable for the strong and hence also the weak operator topology. It may be worth noting that for $X$ a Banach space it is known that $L^{1}\left(P_{T}\right)$ coincides with $L^{\infty}\left(P_{T}\right)$ as a vector space. Accordingly, $L^{\infty}\left(P_{T}\right)$ is always $\tau\left(P_{T}\right)$-separable. Of course, it is rarely separable for the $P_{T}$-essential sup-norm topology given by

$$
\|f\|_{\infty}=\inf \left\{\left\|f \chi_{E}\right\|_{\infty} ; E \in \Sigma, P_{r}(E)=I\right\}, \text { for } f \in L^{\infty}\left(P_{T}\right) .
$$

To treat operator algebras generated by arbitrary complete and $\sigma$-complete Boolean algebras of projections (by realizing the Boolean algebra as the range of a spectral measure) it is necessary to consider $\sigma$-algebras more general than $\mathscr{B}(\mathbb{C})$. The results of this section are formulated for arbitrary operator-valued measures, not just spectral measures.

An operator-valued measure is any set function $P: \Sigma \rightarrow L_{s}(X)$, with domain a $\sigma$-algebra of subsets of some set $\Omega$, which is $\sigma$-additive. The topology of $L_{s}(X)$ is generated by the seminorms

$$
q_{x}: T \mapsto q(T x), \quad T \in L(X),
$$

for every $x \in X$ and $q \in \mathscr{Q}_{X}$. The continuous dual space of $L_{s}(X)$ consists of all finite linear combinations of functionals of the form

$$
\xi_{x, x^{\prime}}: T \mapsto\left\langle T x, x^{\prime}\right\rangle, \quad T \in L(X),
$$

for arbitrary $x \in X$ and $x^{\prime} \in X^{\prime}$. For each $x \in X$, let $P x: \Sigma \rightarrow X$ denote the vector measure $P x: E \mapsto P(E) x$, for $E \in \Sigma$.

The main question is the connection between the separability of the operator-valued measure $P$ and that of the family of (generally simpler) $X$-valued measures $P x, x \in X$, from which $P$ is synthesized.

Proposition 4. Let $X$ be a lcs and $P: \Sigma \rightarrow L_{s}(X)$ be a measure. If $P$ is separable, then each induced $X$-valued measure $P x: \Sigma \rightarrow X, x \in X$, is also separable. If in addition, $X$ is metrizable, then each space $L^{1}(P x), x \in X$, is $\tau(P x)$-separable.

Proof. Fix $x \in X$. Since each $P$-null set is also $P x$-null, it follows that the natural map $\Phi: \Sigma(P) \rightarrow \Sigma(P x)$ which sends the $P$-equivalence class, $[E]_{P}$, of $E \in \Sigma$, to the $P x$-equivalence class $[E]_{P x}$ is well-defined and onto. Since the continuous image of a separable space is separable it suffices to show that $\Phi$ is continuous.

A typical $\tau(P x)$ semi-metric is of the form

$$
d_{q}(E, F)=\sup \left\{\left|\left\langle P x, x^{\prime}\right\rangle\right|(E \Delta F) ; x^{\prime} \in U_{q}^{0}\right\}, \quad E, F \in \Sigma,
$$

where $q \in \mathscr{Q}_{X}$ and $\left\langle P x, x^{\prime}\right\rangle$ is the $\mathbb{C}$-valued measure $\left\langle P x, x^{\prime}\right\rangle: E \mapsto\left\langle P(E) x, x^{\prime}\right\rangle$, for $E \in \Sigma$. A direct calculation shows that, for every $x^{\prime} \in U_{q}^{0}$, the functional $\xi_{x, x^{\prime}}$ given by (9) belongs to the polar set $U_{q_{x}}^{0}$, where $q_{x}$ is the seminorm (8). Since the measures $\left\langle P, \xi_{x, x^{\prime}}\right\rangle$ and $\left\langle P x, x^{\prime}\right\rangle$ coincide, it follows that the right-hand-side of (10) does not exceed $\sup \left\{|\langle P, \xi\rangle|(E \Delta F) ; \xi \in U_{q_{x}}^{0}\right\}=d_{q_{x}}(E, F)$; that is, we have the inequalities $d_{q}(E, F) \leq$ 
$d_{q_{x}}(E, F)$, for $E, F \in \Sigma$, for every $q \in \mathscr{Q}_{X}$. Since each $q_{x}$ is continuous it follows that $d_{q_{x}}$ is one of the semi-metrics generating $\tau(P)$. This shows that $\Phi$ is continuous.

The statement concerning the separability of each space $L^{1}(P x), x \in X$ (in the event that $X$ is metrizable), follows from Proposition 1.

In practice, a converse statement to that of Proposition 4 would be more useful. Unfortunately, no such statement is valid, even for $X$ a "nice" space (eg. a Hilbert space).

EXAMPLE 2. We exhibit an operator-valued measure $P: \Sigma \rightarrow L(X)$ such that

(i) $P$ is not a separable measure,

(ii) $L^{\prime}(P)$ is not a separable space, but

(iii) each measure $P x, x \in X$, is separable and each space $L^{1}(P x)$ is separable.

Indeed, let $X=l^{2}(\Omega)$, where $\Omega$ is a set with $c<\#(\Omega)$, and let $\Sigma$ be the $\sigma$-algebra of all subsets of $\Omega$. For each $E \in \Sigma$, let $P(E) \in L(X)$ denote the operator in $X$ of pointwise multiplication by $\chi_{E}$; here we interpret elements of $X$ as functions $x: \Omega \rightarrow \mathbb{C}$ such that $\sum_{\omega \in \Omega}|x(\omega)|^{2}<\infty$. Then $P: \Sigma \rightarrow L(X)$, so defined, is an operator-valued (even spectral) measure.

Fix $x \in X$. Then $P x$ is the $X$-valued measure $P x: E \mapsto x \chi_{E}$, for $E \in \Sigma$. The space $L^{1}(P x)$ can be identified with all functions $\psi: \Omega \rightarrow \mathbb{C}$ for which the product function $\psi x$ belongs to $X$, with integrals given by $\int_{E} \psi d P x=\psi x \chi_{E}$, for $E \in \Sigma$. Since $\mathscr{Q}_{X}$ consists of a single norm, denoted by $\|\cdot\|_{2}$, the space $L^{1}(P x)$ is a normed space with norm $\|.\|_{x}$ given by

$$
\|\psi\|_{x}=\sup \left\{\int_{\Omega}|\psi| d\left|\left\langle P x, x^{\prime}\right\rangle\right| ;\left\|x^{\prime}\right\|_{2} \leq 1\right\}, \quad \psi \in L^{\prime}(P x) .
$$

We have used the formula (2) and identified $X^{\prime}$ with $X$. But, a direct calculation shows that $\left|\left\langle P x, x^{\prime}\right\rangle\right|$ is the measure $E \mapsto \Sigma_{\omega \in \Omega} \chi_{E}(\omega)\left|x(\omega) x^{\prime}(\omega)\right|$, for $E \in \Sigma$, where the sum consists of countably many terms (since $x x^{\prime} \in l^{\prime}(\Omega)$ implies that there are only countably many points $\omega \in \Omega$ for which $\left.x(\omega) x^{\prime}(\omega) \neq 0\right)$. It follows from this fact and (11) that $\|\psi\|_{x}=\|x \psi\|_{2}$, for $\psi \in L^{1}(P x)$. Now, $x \in X$ implies that $Z(x)=\{\omega \in \Omega ; x(\omega) \neq 0\}$ is a countable set. Furthermore, the metric $d_{x}$ determining the topology $\tau(P x)$ is given by

$$
d_{x}(E, F)=\left\|\chi_{E}-\chi_{F}\right\|_{x}=\left\|\left(\chi_{E}-\chi_{F}\right) x\right\|_{2}, \quad E, F \in \Sigma .
$$

Since any function $h: \Omega \rightarrow \mathbb{C}$ such that $Z(h) \cap Z(x)=\phi$ is $P x$-null, the set (of equivalence classes) $\Sigma(P x)$ is countable. Accordingly, $P x$ is a separable measure. By Proposition 1 also $L^{1}(P x)$ is separable. This establishes (iii).

As a linear space we can identify $L^{1}(P)$ with the space $l^{\infty}(\Omega)$. Indeed, every $f \in l^{\infty}(\Omega)$ has indefinite integral given by $E \mapsto \int_{E} f d P, E \in \Sigma$, where $\int_{E} f d P \in L(X)$ is the operator in $X$ of pointwise multiplication by $\chi_{E} f$. We consider an equivalent family of seminorms generating the topology $\tau(P)$; see [6; Ch. II Sections 1-2]. Recalling that $\mathscr{2}_{X}=\left\{\|\right.$. $\left.\|_{2}\right\}$ this family of seminorms can be specified as

$$
\|f\|_{x, 2}=\sup \left\{\left\|\left(\int_{E} f d P\right) x\right\|_{2} ; E \in \Sigma\right\}=\|x f\|_{2}, \quad f \in L^{1}(P),
$$

for every $x \in X$. 
Let $Y=l^{\infty}(\Omega)$, equipped with the topology of pointwise convergence on $\Omega$. This is a Ics with topology determined by the seminorms $\varphi \mapsto|\varphi(\omega)|, \varphi \in Y$, for each $\omega \in \Omega$. Given $\omega \in \Omega$, the element $e_{\omega}=\chi_{\{\omega\}}$ of $X$ satisfies

$$
\left\|e_{\omega} f\right\|_{2}^{2}=\sum_{s \in \Omega}\left|e_{\omega}(s) f(s)\right|^{2}=|f(\omega)|^{2}, \quad f \in L^{1}(P),
$$

and so (12) shows that the identity map from $L^{1}(P)$ onto $Y$ is continuous. Now, $Y$ is a dense subspace of the lcs $Z=\mathbb{C}^{\Omega}$, equipped with the pointwise convergence topology on $\Omega$ or, equivalently, the product topology. Since $Z$ is non-separable (as $c<\#(\Omega)$ ) it follows that $Y$, hence also $L^{1}(P)$, cannot be separable. This is (ii). Then (i) follows from Proposition 1.

Acknowledgement. I wish to thank Drs Susumu Okada and David Fremlin for valuable discussions on this topic.

Addendum. Shortly before the proofs of this paper arrived Dr D. Fremlin (private communication) showed that the answer to Question (ii) of Remark 5 is negative. Indeed, the seminorms $q(m)$, given by (2), are Riesz seminorms. Accordingly, if $\mathscr{F}$ is dense in $L^{\prime}(m)$ and $A(f)=\left\{\omega ;|f(\omega)-1| \leq \frac{1}{2}\right\}$ then, for $\mathbb{B}$-valued $f$, we have

$$
q(m)\left(\chi_{E}-\chi_{A(f)}\right) \leq 2 q(m)\left(\chi_{E}-f\right)
$$

for every $E \in \Sigma, f \in \mathscr{F}$ and continuous seminorm $q$ in $X$. It follows (even for $\mathbb{C}$-valued $f$ ) that $\left\{\chi_{A(f)} ; f \in \mathscr{F}\right\}$ is dense in $\Sigma(m)$. Accordingly, Proposition 1 can be improved as follows.

Proposition 1A. Let $X$ be a Hausdorff, sequentially complete lcs. Then $m$ is separable if and only if $L^{1}(m)$ is separable.

\section{REFERENCES}

1. W. G. Bade, On Boolean algebras of projections and algebras of operators, Trans. Amer. Math. Soc. 80 (1955), 345-359.

2. P. G. Dodds and W. Ricker, Spectral measures and the Bade reflexivity theorem, $J$. Functional Analysis 61 (1985), 136-163. 1964).

3. N. Dunford and J. T. Schwartz, Linear operators I, 2nd Printing (Interscience, New York,

4. E. Hewitt and K. Stromberg, Real and abstract analysis, Graduate Texts in Math., Vol. 25 (3rd Edition) (Springer-Verlag, 1975).

5. I. Kluvánek, The extension and closure of vector measures, in Vector and operator-valued measures and applications, pp. 175-189 (Academic Press, 1973).

6. I. Kluvánek and G. Knowles, Vector measures and control systems (North Holland, Amsterdam, 1976). $75-80$.

7. W. Ricker, Criteria for closedness of vector measures, Proc. Amer. Math. Soc. 91 (1984),

8. A. C. Zaanen, Integration (North Holland, Amsterdam, 1967).

School of Mathematics

University of New South Wales

KENSINGTON 2033

Australia 